\title{
Negative Mood and Food Craving Strength Among Women with Overweight: Implications for Targeting Mechanisms Using a Mindful Eating Intervention
}

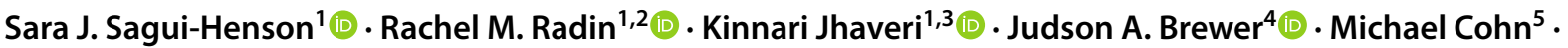 \\ Wendy Hartogensis ${ }^{1}$ (D) . Ashley E. Mason ${ }^{1,2}$ (D)
}

Accepted: 10 September 2021 / Published online: 24 September 2021

(c) The Author(s), under exclusive licence to Springer Science+Business Media, LLC, part of Springer Nature 2021

\begin{abstract}
Objectives When experiencing negative mood, people often eat to improve their mood. A learned association between mood and eating may cultivate frequent food cravings, detracting from health goals. Training in mindful eating may target this cycle of emotion-craving-eating by teaching individuals to manage urges when experiencing negative mood. We examined the impact of a mobile mindful eating intervention on the link between negative mood and food cravings among overweight women.

Methods In a single-arm trial, participants $\left(n=64, M\right.$ age $=46.1$ years, $M$ BMI $\left.=31.5 \mathrm{~kg} / \mathrm{m}^{2}\right)$ completed ecological momentary assessments of negative mood and food cravings 3 times/day for 3 days pre- and post-intervention, as well as 1-month post-intervention. Using multilevel linear regression, we compared associations between negative mood and food craving strength at pre- vs. post-intervention (model 1) and post-intervention vs. 1-month follow-up (model 2).

Results In model 1, negative mood interacted with time point $(\beta=-.20, S E=.09, p=.02,95 \%$ CI [ $-.38,-.03])$ to predict craving strength, indicating that the within-person association between negative mood and craving strength was significantly weaker at post-intervention $(\beta=0.18)$ relative to pre-intervention $(\beta=0.38)$. In model 2 , negative mood did not interact with time point to predict craving strength $(\beta=.13, S E=.09, p=.10,95 \% \mathrm{CI}-.03, .31])$; the association did not significantly differ between post-intervention and 1-month follow-up.

Conclusions Training in mindful eating weakened the mood-craving association from pre- to post-intervention. The weakened association remained at follow-up. Our findings highlight the mood-craving link as a target-worthy mechanism of mindful eating that should be assessed in clinical trials.
\end{abstract}

Trial Registration NCT02694731

Keywords Negative mood $\cdot$ Craving $\cdot$ Meating $\cdot$ Ecological momentary assessment

Ashley E. Mason

Ashley.Mason@UCSF.edu

1 Osher Center for Integrative Medicine, University of California San Francisco (UCSF), 1545 Divisadero Street, 3rd Floor, Ste 301, San Francisco, CA 94115, USA

2 Department of Psychiatry and Behavioral Sciences, UCSF, San Francisco, USA

3 Department of Psychology, University of Southern California, Los Angeles, USA

4 Brown University School of Public Health and Medical School, Providence, USA

5 Google Inc, Mountain View, USA
Food cravings are defined as frequent, intense desires to consume certain types of food (Weingarten \& Elston, 1990). They are common (Hill \& Heaton-Brown, 1994), especially among people attempting to lose weight (Elfhag \& Rossner, 2005; Massey \& Hill, 2012) and with higher body mass index (BMI; Chao et al., 2014; Delahanty et al., 2002). Food cravings are associated with other forms of non-homeostatic (i.e., eating for reasons other than energy needs) such as loss of control over eating, emotional eating, reward-driven eating, and binge eating (Dalton et al., 2013; Hill et al., 1991; Meule et al., 2012). They are considered distinct from physiological or homeostatic eating behavior (i.e., eating in response to hunger cues). Thus, food cravings are an important behavioral target in the context of behavioral weight 
loss interventions (Mason et al., 2018), yet the mechanisms through which we can successfully reduce cravings remain unclear.

Cravings for highly palatable foods are difficult to change because they are deeply rooted in learned behavioral processes (Brewer et al., 2018; Epstein et al., 2007). Specifically, eating in response to negative mood is a conditioned and reinforced response. In this sense, emotional and craving-related eating share the feature of negative affect as a trigger. For example, negative emotions often precede food cravings and dietary lapses follow (Carels et al., 2001). Data also suggest that negative emotions precede binge eating episodes (Haedt-Matt \& Keel, 2011). A key difference between emotional and craving-related eating is the specificity of food consumed; in emotional eating, individuals seek palatable foods in general, whereas in craving-related eating, individuals focus on specifically craved foods (Weingarten \& Elston, 1990). Additionally, people who self-identify as addicted to chocolate report that cravings for chocolate arise more often in response to mood than to hunger (Hetherington \& MacDiarmid, 1993). In these contexts, eating highly palatable food that one craves in response to negative mood may serve the short-term goal of improving mood (Corsica \& Spring, 2008; Ventura et al., 2014), but over time, this learned association between negative mood and food cravings will detract from longer term health goals (e.g., weight loss and metabolic health).

Affective regulation models have been well-validated among adults with binge eating (i.e., consumption of an objectively large amount of food + the experience of loss of control over eating). Binge eating overlaps significantly with craving-related eating (Ng \& Davis, 2013), although researchers have arguably examined binge eating in greater detail given its clinical severity. The affect model of binge eating describes the importance of negative emotional experiences in triggering eating episodes (Arnow et al., 1995; Heatherton \& Baumeister, 1991; Polivy \& Herman, 1993; Telch \& Agras, 1996). Binge eating occurs during times of distress, when individuals lack skills to adaptively cope with affective states, and binge eating becomes the primary means of coping to relieve emotional distress. Empirical data have supported this model, including studies that utilize ecological momentary assessment of affective triggers (Agras \& Telch, 1998; Haedt-Matt \& Keel, 2011; Telch \& Agras, 1996; Whiteside et al., 2007).

From a reinforcement learning perspective, a negative mood may trigger food cravings. Satisfying food cravings by eating the craved food may provide negative reinforcement (i.e., removal of negative mood; Bouton, 2011). A relief in negative mood after eating increases the likelihood of experiencing a food craving and eating in response when experiencing a negative mood (Skinner, 1963). This feedback loop can result in increased non-homeostatic appetite and overeating that serve to regulate mood, but ultimately lead to weight gain and obesity (Singh, 2014). Negative mood may be a driver of food cravings (and in recent data, cravings for sugar-sweetened beverages) as a learned behavior (Falbe et al., 2019). The link between mood and cravings may serve to maintain dysregulated eating behavior during times of distress. Therefore, efforts to manage or reduce craving-driven eating must target the learned cycle between negative mood and food craving.

Mindfulness-based approaches have shown promise as a treatment modality for adults with food cravings. They aim to cultivate a non-judging awareness of experiences in the present moment and promote greater self-regulation (Brown et al., 2007). General mindfulness-based interventions reduce stress reactivity (Hoge et al., 2013) and emotional and binge eating (Sala et al., 2020). Recent cross-sectional work suggests that acting with mindful awareness weakens associations between emotional and binge eating severity (Barnhart et al., 2021).

Training in mindful eating may be an effective way to target these negatively reinforced food cravings by clarifying automatic behavior that follows from a learned association between negative mood and food cravings. Mindful eating involves non-judgmentally paying attention to an eating experience with all five senses and noticing emotional and physical responses before, during, and after eating (Kristeller \& Wolever, 2010). Mindful eating interventions emphasize awareness of emotional triggers of food cravings and the re-learning of adaptive habits. For example, the mindfulness-based eating awareness training (MB-EAT; Kristeller \& Wolever, 2010) program teaches individuals to cultivate greater awareness of emotional states and external triggers (e.g., stress) with the goal of interrupting dysfunctional cycles of emotional eating (Kristeller et al., 2014). Mindfulness-based approaches that specifically incorporate mindful eating techniques have shown promise in reducing binge eating (Godfrey et al., 2015), specifically the frequency and the severity of eating episodes (Kristeller \& Hallett, 1999; Kristeller \& Wolever, 2010; Kristeller et al., 2014).

Training in mindful eating is of particular interest because it may continue to impart benefits after the intervention has ended. For example, across several mindfulness-based interventions tailored for eating behavior, participants often continue improving on outcomes for several weeks or months after completing the program. For example, participants in a combined mindfulness and cognitive behavioral therapy program maintained significant reductions in binge eating and dieting three months post-intervention (Woolhouse et al., 2012). Research using the MB-EAT intervention has also established sustainable effects on eating behavior four months post-intervention (Kristeller et al., 2014).

Although mindful eating interventions generally have a beneficial impact on eating behaviors, we still do not know 
if training in mindful eating exerts its effects by breaking the link between negative mood and food cravings. For example, one large randomized controlled trial that incorporated elements of MB-EAT found significant reductions in the consumption of sweets (Mason, et al., 2016a, b) and reward-driven eating (Mason, et al., 2016a, b). The authors also saw improvements in metabolic health in the intervention (compared to active control) group (Daubenmier et al., 2016). Several other mindful eating interventions report similar results (e.g., Beshara et al., 2013; Jordan et al., 2014). Unfortunately, these interventions were not delivered with a modality that allowed researchers to measure daily-level associations between negative mood and craving, which are critical in identifying the role of this potentially mechanistic link.

Negative mood and food cravings are dynamic processes that fluctuate over time within individuals. The momentary nature of these processes and how they influence each other require assessment methodology that can capture the micro-temporal relationship between variables of interest (Shiffman et al., 2008). Ecological momentary assessment (EMA) examines daily behavior and psychological states of individuals in their natural environment (Stone \& Shiffman, 1994). Using in-the-moment assessment tools by which participants complete ratings about their current state, EMA methods provide prospective and ecologically valid data on participants in their daily living environment. Reporting on affect and behavior close in time to real-world experiences minimizes recall bias and offers improved reliability over retrospective questionnaires and interviews (Bolger \& Laurenceau, 2013). By allowing repeated measures over time, EMA is well-suited to capture variability over time and assess how negative mood and food cravings relate to each other throughout the day (Smyth et al., 2009; Wegner et al., 2002).

Studies utilizing EMA methods have reported links between negative affect and eating (Haedt-Matt \& Keel, 2011). For example, in a study where participants used palmtop computers to answer questions about eating and emotions several times a day, they reported a decrease in positive mood immediately preceding binge eating episodes (Munsch et al., 2012). Another study asked participants who endorsed binge eating disorder to rate their mood on 32 randomized occasions per day in addition to before, during, and after eating, using a portable minicomputer (Hilbert $\&$ Tuschen-Caffier, 2007). Self-reported mood was significantly more negative preceding binge eating episodes than it was prior to normal eating episodes. As demonstrated by these studies, EMA proved a useful assessment tool to assess the assocation between mood and cravings throughout the day (Haedt-Matt \& Keel, 2011). However, no studies have tested daily mood as a predictor of food cravings within the context of a mindful eating intervention.
Mason and colleagues (2018) developed a mobile mindful eating intervention that targets links between negative mood and cravings, called Eat Right Now and daily assessment methods that would allow for exploration of mechanism. The intervention trains participants in mindful eating and offers specific eating and food craving meditations (Mason et al., 2018). The program offers participants tools to aid in mindfully "riding out" food cravings as they occur in the context of emotional triggers. In a single-arm trial of Eat Right Now, participants' food cravings and overeating behavior were significantly reduced one month following intervention completion (1-month follow-up; Mason et al., 2018). Elucidating how this mindful eating intervention impacts a link between negative mood and food cravings at the daily level would clarify the importance of targeting this link using mindfulness interventions. Specifically, by learning whether this mindful eating intervention weakens this link, researchers can better tailor mindful eating interventions to capitalize on daily fluctuations in mood.

These analyses used data collected via EMA to test whether individuals report stronger food cravings when they also experience greater negative mood, and if this withinperson association changes following a mindful eating intervention. We hypothesized that, using multilevel models at the within-person level, (1) greater negative mood would be associated with stronger food cravings before the intervention (model 1), (2) this association would be significantly weaker at post-intervention than at pre-intervention (model 1 ), and (3) this association would be significantly weaker one-month post-intervention (follow-up) than at post-intervention (model 2).

\section{Method}

\section{Participants}

Participants learned of the study through social media outlets, UCSF campus-wide email digests, and targeted physical letters to UCSF patients. We invited prospective participants to complete a web-based screening survey (www.qualtrics. com) and a pre-intervention text message assessment period to verify eligibility. Eligible participants were women aged at least 18 years with a BMI of 25 or greater, did not have diabetes (per self-report), had a smartphone operating iOS or Android, experienced food cravings most days of the week, and ate in response to cravings more often than desired (selfreport), responded to at least 7 of 9 text messages during the pre-intervention eligibility period, and reported at least 3 instances of craving-related eating in these text message assessments. We opted for these inclusion criteria to ensure that we recruited the population of interest: Individuals who experience frequent food cravings that they find problematic. 
The initial target sample of 105 participants (Mason et al., 2018) was based off of a previously completed power analysis, estimated to predict a $30 \%$ reduction in frequency of craving-related eating.

On average, participants were $47.8 \pm 14.4$ years old and had a BMI of $30.8 \pm 4.1 \mathrm{~kg} / \mathrm{m}^{2}$. Of the participants, $9.4 \%$ identified as Asian or Asian American, 4.7\% identified as non-Hispanic Black or African American, 72\% identified as non-Hispanic White or Caucasian, $10.9 \%$ identified as Hispanic or Latino/a, and 3.1\% identified as a non-Hispanic member of another racial/ethnic background.

\section{Procedures}

Study Design We analyzed data from the Eat Right Now trial (Mason et al., 2018), which deployed an app-based mindful eating intervention (ClinicalTrials gov registration: NCT02694731). This was a single-arm, unmasked trial (phase IIA) that assessed participants at pre- and postintervention, and one-month post-intervention completion. Participants completed a 28-lesson self-paced smartphonedelivered intervention targeting craving-related eating using mindful eating practices and completed daily text message assessments of mood and food cravings at pre-intervention, post-intervention, and 1-month follow-up. Study design and methods are reported in detail elsewhere (Mason et al., 2018). The University of California, San Francisco (UCSF) Institutional Review Board, approved all study procedures. All participants provided informed consent.

Screening To ensure that we enrolled participants who experienced problematic food cravings, we first asked prospective participants two self-report questions, "Do you have food cravings most days of the week?" and "Do you eat in response to food cravings more than you wish you did?" Individuals responded yes or no to these questions. If they responded yes to both, we sent them 3 SMS text messages each day for 3 days asking about craving-related eating. To be eligible, participants needed to report at least 3 instances of craving-related eating during this period. These procedures allowed us to ensure that we were recruiting participants who experienced problematic food cravings that they wished to change. We collected weight and height from eligible participants at the baseline visit to calculate BMI. Eligible participants attended an in-person baseline assessment at UCSF where they completed self-report measures and height and weight assessments to complete eligibility determination.

Study Participation If determined eligible at the in-person baseline visit, participants installed the mindful eating intervention. Participants completed the intervention at their own pace (see "Intervention Stucture and Content") and on average completed the intervention in 47 days. Participants completed EMA assessments via SMS text messages three times per day over three days over the course of one week pre-intervention, one week immediately post-intervention, and one week at 1-month follow-up (see "Measures"). Participants completed an in-person post-intervention assessment at which they again completed self-report measures and height and weight assessments. Participants completed self-report measures online at 1-month follow-up.

Intervention Structure and Content Eat Right Now is a theory-driven, self-paced, smartphone-delivered mindful eating intervention targeting the experience of cravings and craving-related eating. Participants completed lessons over a minimum of 28 days, with one new lesson becoming available each day. Participants accessed one 5- to 10-min module per day and each module contained a video lecture with animations and straightforward guidance as to how to practice mindful eating principles. Modules for future days were locked to prevent skipping, yet participants could review previous modules with unlimited access. The intervention was self-paced, which allowed participants to spend additional time consolidating their new knowledge, practicing mindfulness skills, or using the in-app tools prior to starting the next module.

The intervention content focused on mindful eating, as well as habit formation, behavioral management, existing with cravings, loving-kindness, curiosity, strategizing, and reinforcing concepts. Course materials taught participants to attend to three aspects of eating: why, what, and how. Why they eat, which included emotional triggers unrelated to homeostatic hunger; What types of food are most likely to lead to and reinforce cravings; and How to eat with mindful attention to physiological cues in the presence of difficult emotions. The ERN intervention presented content in the form of brief daily videos that narrate key concepts of mindful eating (see Mason et al., 2018, for overview of the ERN intervention).

Participants could also access to tools to help them manage food cravings. For instance, the "Stress Test" consisted of short mindfulness exercise that encouraged participants to check in with their bodies and observe and current feeling states. They received feedback about whether symptoms they endorsed were most consistent with stress, a habit-driven urge to eat, or homeostatic hunger. The app then provided relevant suggestions (e.g., to eat mindfully, or use a mindfulness exercise to ride out a craving). Another tool was the "Want-O-Meter" button, which guided individuals through a craving. Based on individuals's responses, they were then funneled to additional tools, such as the RAIN (Recognize, Accept, Investigate, and Note) exercise, which aims to teach individuals how to mindfully respond to food cues, affective states, and food cravings and promotes separating the 
experiences of food cravings and eating in response to them. Participants could choose to eat with awareness in response to a craving - the app provided a specific mindful eating exercise.

Participants could set reminders to check in with their hunger and emotional state, to use mindfulness skills, and to track progress towards goals. After completing 28 modules, all tools and modules remained available indefinitely to participants to support ongoing mindful eating. We designed this intervention to help individuals incorporate the didactic content and mindful eating practices into their everyday activities and eating routines.

\section{Measures}

EMA Measures We collected EMA measures over one-week periods during eligibility, at pre- and post-intervention, and at 1-month follow-up. We sent participants SMS text messages at 11:30 AM, 4:30 PM, and 9:00 PM for 3 days spread over the course of each week ( 2 weekdays and 1 weekend day). We opted for two weekdays and one weekend day in an effort to assess participants on workdays and non-workdays, and we selected these times of day so as to be outside of somewhat typical mealtimes (before lunch, before dinner, and after dinner). Each text message contained a link (www. qualtrics.com) to a short web-based survey. EMA measures included the following:

Negative Mood We measured negative mood using the following question: "What is the worst you've felt in the past hour?" Participants responded on a visual analog scale ranging from 0 to 100 with three qualitative anchors: very bad (left anchor), okay (middle anchor), very good (right anchor). We rescaled raw scores to a 1-10 interval and recoded this variable so higher scores indicated greater negative mood (Bolger \& Laurenceau, 2013).

Food Craving Strength We measured food cravings using the following question: "Have you had a craving for a food or drink in the past hour?" Participants responded on a visual analog scale ranging from 0 to100 with three qualitative anchors: not at all (left anchor), somewhat (middle anchor), very much (right anchor). We rescaled raw scores to a 1-10 interval with higher scores indicating greater food cravings.

Covariates Because of previously reported associations between age, race/ethnicity, and food cravings (Abdella et al., 2019; Chao et al., 2014), we adjusted for these variables in adjusted analyses (results from unadjusted models are presented in Supplementary Material). Participants self-reported their age (years) and their ethnicity (Hispanic or Latino/Latina (yes/no) plus racial category that best described themselves from pre-selected options: Asian or Asian American, Black or African American, White, Native American or Eskimo, Pacific Islander, Hispanic or Latino/ Latina, or Other. We collapsed race and ethnicity questions into a dichotomous variable coded as 0 (non-White) and 1 (non-Hispanic White).

\section{Data Analyses}

Variable Preparation To facilitate interpretation of the intercept in the analyses, we grand-mean centered (Singer \& Willett, 2003) scores on the negative mood variable by subtracting the mean across participants and time points from each score. Because we collected repeated assessments per participant, data could vary within person (across assessments) or could be averaged to a score that varies between people. To focus on the within-person variation, we prepared the negative mood scores for multilevel analysis by separating this variation. We created a between-person variable (between negative mood) by averaging the grand-mean centered scores across time points (i.e., each participant had one negative mood score for pre- and post-intervention, and follow-up). We created a within-person variable (within negative mood) by subtracting the between negative mood scores from the grand-mean centered scores. This removed the portion of the grand-mean centered scores that differed between participants and left us with the portion that differed by assessment (within person). While we assumed between- and within-person variances in food craving strength, it was not necessary to manually calculate, as SPSS decomposes the variance of the dependent variable by default into betweenand within-person components. Thus, the model coefficients represent associations among negative mood and food craving strength at each level separately (i.e., the between negative mood coefficients show associations among betweenperson negative mood and between-person food cravings). We examined data missingness, frequencies, distributions, skewness, and kurtosis for each study variable.

Data Selection Prior evidence from this trial suggested that completing the intervention within three months conferred greater clinical improvement. Those who completed the intervention within three months ("timely completers"), lost approximately 2 pounds $(t=2.41, p=0.019)$, and the faster they completed the intervention, the weaker they reported their cravings to be (Mason et al., 2018). Thus, we examined only the 64 participants (82\%) who completed the intervention within three months ("timely completers").

Statistical Analyses We tested our hypotheses that (1) greater negative mood would be associated with stronger food cravings before a mindful eating intervention and that this association would be significantly weaker after the 
intervention (model 1; pre-post), and that (2) the positive association between greater negative mood and stronger food cravings at one-month post-intervention would be further weakened relative to post-intervention (model 2; postfollow-up). To test these hypotheses, we used multilevel linear regression models (linear mixed modeling command in SPSS 25.0; IBM Corp, 2017; Singer \& Willett, 2003). We analyzed EMA data within a hierarchical structure: we clustered multiple observations (level 1) within participants (level 2). Before analyses, we examined the ICC value for food cravings (0.14), which indicated support for multilevel analysis (Singer \& Willett, 2003).

Our models specified a within-person association between negative mood and food cravings that we predicted would be weaker at post-intervention when compared with pre-intervention and weaker at follow-up when compared with post-intervention. Thus, we conducted two unadjusted models: one comparing pre-intervention (coded as 0) and post-intervention (coded as 1), and one comparing postintervention (coded as 0 ) and one-month post-intervention (coded as 1). In adjusted models, we included race/ethnicity $(0=$ non-White, $1=$ non-Hispanic White) and age (mean centered) as covariates in each model.

In model 1 (pre-post), the parameters of interest were within negative mood (within-person association between negative mood and food cravings at pre-intervention) and the interaction between within negative mood and time point (the difference in the negative mood-food craving slope between pre- and post-intervention time points). In model 2 (post-follow-up), the parameter of interest was the interaction among within negative mood and time point (the difference in the negative mood-food craving slope between postintervention and follow-up time points). For these analyses, we allowed intercepts and the within negative mood slope to vary randomly. We assumed the presence of autoregressive effects from one assessment to the next, and therefore included a first-order autoregressive term (AR, [1]) in the repeated measures model.

\section{Results}

\section{Completeness}

Participants completed up to 27 assessments ( 3 assessments per day for 3 days pre-intervention, post-intervention, and at follow-up). There were 107 (6.6\%) observations missing, for a total of 1621 EMA observations. Less than $7 \%$ of cases were missing data on the analyzed variables, and Little's Missing Completely at Random Test (Little, 1988) demonstrated that the data were missing completely at random, $\chi^{2}=1847.59$ (1881), $p=0.70$. Therefore, we used listwise deletion in our analyses. We identified multivariate outliers, with the use of Mahalanobis distance, in less than $0.11 \%$ of all data points. Although there were no apparent time trends in the data based on visual inspection, we included in the model a centered version of time (number of assessments).

\section{Univariate Statistics and Bivariate Correlations}

Table 1 includes the mean, standard deviation, skewness, kurtosis, and bivariate correlations for negative mood and food cravings at each time point. Descriptive statistics across participants and time points for negative mood were $M=4.85, S D=2.14$, and range $1-10$ and for food cravings were $M=4.85, S D=2.92$, and range 1-10. Descriptive statistics at each time point indicated that, on average,
Table 1 Univariate statistics and bivariate correlations among negative mood and food cravings across participants and time points

\begin{tabular}{|c|c|c|c|c|c|c|}
\hline & \multicolumn{3}{|c|}{ Negative mood } & \multicolumn{3}{|c|}{ Food cravings } \\
\hline & Pre & Post & FU & Pre & Post & FU \\
\hline Negative mood pre & - & & & & & \\
\hline Negative mood post & $.15^{* *}$ & - & & & & \\
\hline Negative mood FU & $.13^{* *}$ & $.24 * *$ & - & & & \\
\hline Food cravings pre & $.28 * *$ & .04 & .07 & - & & \\
\hline Food cravings post & .01 & $.14 * *$ & .06 & $.15 * *$ & - & \\
\hline Food cravings FU & $-.12 *$ & .08 & $.19^{* *}$ & $.12 *$ & $.28 * *$ & - \\
\hline Mean & 5.17 & 4.78 & 4.58 & 5.54 & 4.56 & 4.43 \\
\hline$S D$ & 2.19 & 2.11 & 2.07 & 2.95 & 2.88 & 2.78 \\
\hline Skewness & -0.05 & 0.25 & 0.36 & -0.17 & 0.18 & 0.11 \\
\hline Kurtosis & -0.48 & -0.48 & -0.19 & -1.19 & -1.24 & -1.36 \\
\hline
\end{tabular}

Note. $N=64$ participants, 27 assessments ( 9 pre-intervention, 9 post-intervention, 9 follow-up), 1621 observations. Statistics are presented in aggregate. Pre, pre-intervention; Post, post-intervention; FU, onemonth follow-up

${ }^{*} p<.01, * * p<.001$ 
participants reported moderate negative mood and food cravings based on the sample range. Generally, Pearson correlations ranged from small to medium (e.g., 0.01 to 0.28 ) with most correlations being small. Correlations between negative mood and food cravings at concurrent time points were positive and statistically significant at all time points, with the strength decreasing at post-intervention. Importantly, all univariate measures of skewness and kurtosis were between -2 and +2 , which permitted the use of normal theory maximum likelihood estimation.

\section{Hypothesis Testing}

\section{Model 1: Associations Among Negative Mood and Food Cravings from Pre- to Post-intervention}

Within-person negative mood was significantly related to food cravings at pre-intervention ( $\beta=0.38,95 \%$ CI [0.23, 0.53 ], $p<0.001$; see Table 2) such that greater negative mood was associated with stronger food cravings. Consistent with our hypothesis, there was a significant interaction among negative mood and time point (within negative mood by time point under fixed effects in Table 2). The interaction term $(\beta=-0.20,95 \%$ CI $[-0.38,-0.03], p=0.021)$ represents the difference in slopes from pre- to post-intervention and shows that the association among negative mood and food cravings was significantly lower at post-intervention.

Specifically, at the within-person level, the post-intervention slope was $\beta=0.18$ (calculated by adding the interaction estimate from the within negative mood estimate). Note that because the analysis model included the within- and between-person forms of negative mood, results for this hypothesis cannot be artifacts of between-person differences in average negative mood over the pre- and post-intervention EMA periods. Figure 1 panel A shows fitted regression lines for each participant at pre- and post-intervention with grey fitted lines for the average participant. Visual inspection of the grey lines indicated that the slope for the association between negative mood and food cravings is less steep at post-intervention than at pre-intervention.

As seen in Fig. 1 (panel A), there was substantial between-person variability in slopes and intercepts at both
Table 2 Parameter estimates for multilevel model 1: food craving as a function of negative mood at pre- and postintervention

\begin{tabular}{|c|c|c|c|c|c|c|}
\hline \multirow[t]{2}{*}{ Fixed effects (intercept, slope) } & \multirow[t]{2}{*}{ Estimate } & \multirow[t]{2}{*}{$(S E)$} & \multirow[t]{2}{*}{$t^{a}$} & \multirow[t]{2}{*}{$p^{b}$} & \multicolumn{2}{|l|}{$95 \%$ CI } \\
\hline & & & & & Lower & Upper \\
\hline Intercept & 5.23 & $(0.29)$ & 17.92 & $<.001$ & 4.65 & 5.81 \\
\hline Within negative mood & 0.38 & $(0.08)$ & 4.96 & $<.001$ & 0.23 & 0.53 \\
\hline Time point (pre-post) ${ }^{\mathrm{c}}$ & -0.89 & $(0.19)$ & -4.77 & $<.001$ & -1.26 & -0.52 \\
\hline Within negative mood by time point & -0.20 & $(0.09)$ & -2.31 & .021 & -0.38 & -0.03 \\
\hline Between negative mood & 0.44 & $(0.21)$ & 2.11 & .038 & 0.03 & 0.85 \\
\hline Between negative mood by time point & -0.38 & $(0.21)$ & -1.82 & .070 & -0.80 & 0.03 \\
\hline Non-Hispanic white ${ }^{\mathrm{d}}$ & 0.28 & $(0.33)$ & 0.84 & .40 & -0.39 & 0.95 \\
\hline Age & -0.00 & $(0.01)$ & -0.02 & .98 & -0.02 & 0.02 \\
\hline \multirow[t]{2}{*}{ Random effects ([co-]variances) } & Estimate & $(S E)$ & $z$ & $p^{b}$ & $95 \% \mathrm{CI}^{\mathrm{e}}$ & \\
\hline & & & & & Lower & Upper \\
\hline \multicolumn{7}{|l|}{ Level 2 (between person) } \\
\hline Intercept & 0.78 & $(0.25)$ & 3.16 & .002 & 0.42 & 1.45 \\
\hline Within negative mood & 0.13 & $(0.05)$ & 2.79 & .005 & 0.06 & 0.26 \\
\hline Intercept and within negative mood & 0.02 & $(0.07)$ & 0.22 & .83 & -0.13 & 0.16 \\
\hline \multicolumn{7}{|l|}{ Level 1 (within person) } \\
\hline Residual & 6.94 & $(0.34)$ & 20.66 & $<.001$ & 6.31 & 7.63 \\
\hline Autocorrelation & 0.14 & $(0.04)$ & 3.88 & $<.001$ & 0.07 & 0.21 \\
\hline
\end{tabular}

Note. $N=64$ participants, 18 assessments (9 pre-intervention, 9 post-intervention), 1074 observations. Pre, pre-intervention; Post, post-intervention

${ }^{a}$ We took a conservative approach to specifying degrees of freedom; these were based on the number of participants $(N=64)$ rather than the number of observations $(N=1074)$

${ }^{\mathrm{b}}$ All $p$-values are two-tailed except in the case of variances, where one-tailed $p$-values are used (because variances are constrained to be non-negative)

${ }^{\mathrm{c}}$ Time point is coded 0 for pre-intervention and 1 for post-intervention

${ }^{\mathrm{d}}$ Non-Hispanic white is coded 0 for participants who self-identified as not white and 1 for participants who self-identified as non-Hispanic white

${ }^{e}$ Confidence intervals (CI) for variances were computed using the Satterthwaite method (see Littell et al., 2006) 


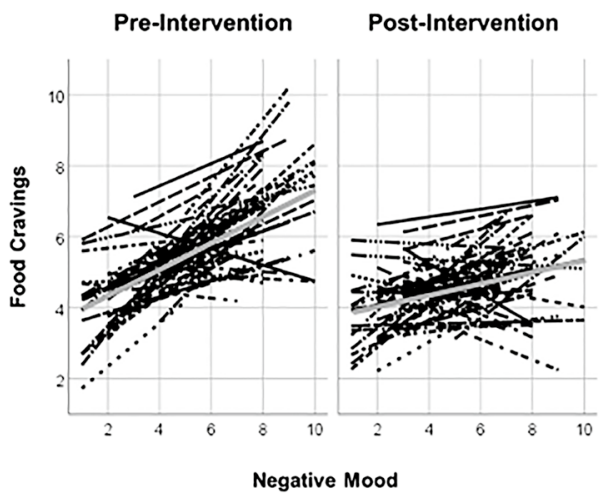

Panel A

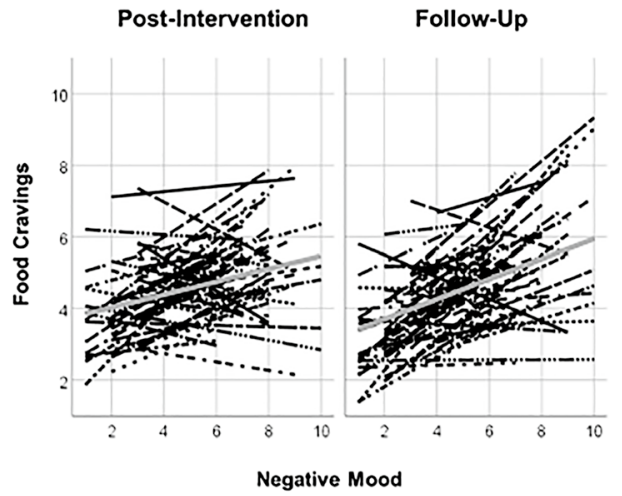

Panel B

the mixed effects model 1. Panel B: Spaghetti plots of average (grey) and participant-specific (black) regression lines for food cravings as a function of negative mood at post-intervention (left) and follow-up (right). The graphs display fitted estimates from the mixed effects model 2

negative mood and food cravings was not significantly different at follow-up.

Figure 1 panel B shows fitted regression lines for each participant at post-intervention and follow-up with grey fitted lines for the average participant. Visual inspection of the grey lines supports the conclusion that the slope for the association between negative mood and food cravings is not significantly different at follow-up compared to post-intervention.

Figure 1 panel B again shows substantial between-person variability in slopes and intercepts at both post-intervention and follow-up. The lower panel of Table 2 presents numerical estimates and statistical tests of that variability. These are reported as variances and covariances. Expressed as standard deviation, the variation for within-person negative mood slopes is again $\sqrt{0.13}=0.36$ units, which, assuming a normal distribution in the population, implies that approximately $95 \%$ of the population are within \pm 2.0 $* 0.36= \pm 0.72$ units of the typical value at post-intervention and follow-up. Accordingly, the model predicts that participants at post-intervention have a $95 \%$ range of slopes of $0.19 \pm 0.72=-0.53$ to 0.91 . The model predicts that participants at follow-up have a similar range from $0.33 \pm 0.72=-0.39$ to 1.05 . The unadjusted model yielded identical patterns of statistical significance (Table S2 in Supplementary Material).

\section{Discussion}

We examined changes in the association between negative mood and food cravings before and after a smartphonedelivered mindful eating intervention. We hypothesized 
Table 3 Parameter estimates for multilevel model 2: food craving as a function of negative mood at post-intervention and follow-up

\begin{tabular}{|c|c|c|c|c|c|c|}
\hline \multirow[t]{2}{*}{ Fixed effects (intercept, slope) } & \multirow[t]{2}{*}{ Estimate } & \multirow[t]{2}{*}{$(S E)$} & \multirow[t]{2}{*}{$t^{a}$} & \multirow[t]{2}{*}{$p^{b}$} & \multicolumn{2}{|l|}{$95 \% \mathrm{CI}$} \\
\hline & & & & & Lower & Upper \\
\hline Intercept & 4.07 & $(0.31)$ & 13.33 & $<.001$ & 3.46 & 4.68 \\
\hline Within negative mood & 0.19 & $(0.08)$ & 2.44 & .016 & 0.04 & 0.35 \\
\hline Time point (post-FU) ${ }^{\mathrm{c}}$ & -0.12 & $(0.16)$ & -0.74 & .46 & -0.44 & 0.20 \\
\hline Within negative mood by time point & 0.14 & $(0.09)$ & 1.63 & .10 & -0.03 & 0.31 \\
\hline Between negative mood & 0.14 & $(0.21)$ & 0.67 & .51 & -0.28 & 0.56 \\
\hline Between negative mood by time point & -0.26 & $(0.18)$ & -1.40 & .16 & -0.62 & 0.10 \\
\hline Non-Hispanic white ${ }^{\mathrm{d}}$ & 0.67 & $(0.35)$ & 1.92 & .060 & -0.03 & 1.39 \\
\hline Age & 0.00 & $(0.01)$ & 0.01 & .99 & -0.02 & 0.02 \\
\hline \multirow[t]{2}{*}{ Random effects ([co-]variances) } & \multirow[t]{2}{*}{ Estimate } & \multirow[t]{2}{*}{$(S E)$} & \multirow[t]{2}{*}{$z$} & \multirow[t]{2}{*}{$p^{b}$} & $95 \% \mathrm{CI}^{\mathrm{e}}$ & \\
\hline & & & & & Lower & Upper \\
\hline \multicolumn{7}{|l|}{ Level 2 (between person) } \\
\hline Intercept & 1.08 & $(0.27)$ & 3.95 & $<.001$ & 0.66 & 1.77 \\
\hline Within negative mood & 0.13 & $(0.04)$ & 3.00 & .003 & 0.07 & 0.25 \\
\hline Intercept and within negative mood & 0.01 & $(0.08)$ & 0.14 & .89 & -0.14 & 0.16 \\
\hline \multicolumn{7}{|l|}{ Level 1 (within person) } \\
\hline Residual & 6.18 & $(0.29)$ & 21.52 & $<.001$ & 5.64 & 6.77 \\
\hline Autocorrelation & 0.04 & $(0.04)$ & 1.00 & .32 & -0.03 & 0.10 \\
\hline
\end{tabular}

Note. $N=64$ participants, 18 assessments (9 post-intervention, 9 follow-up), 1077 observations

Post, post-intervention; $F U$, one-month follow-up

${ }^{a}$ We took a conservative approach to specifying degrees of freedom; these were based on the number of participants $(N=64)$ rather than the number of observations $(N=1077)$

${ }^{\mathrm{b}}$ All $p$-values are two-tailed except in the case of variances, where one-tailed $p$-values are used (because variances are constrained to be non-negative)

${ }^{\mathrm{c}}$ Time point is coded 0 for post-intervention and 1 for follow-up

${ }^{\mathrm{d}}$ Non-Hispanic white is coded 0 for participants who self-identified as not white and 1 for participants who self-identified as non-Hispanic white

${ }^{\mathrm{e}}$ Confidence intervals (CI) for variances were computed using the Satterthwaite method (see Littell et al., 2006)

that greater negative mood would be associated with greater craving strength before the intervention and that this association would be significantly weakened at post-intervention, and further weakened at one-month follow-up. In support of our first hypothesis, we found that greater negative mood was significantly associated with greater food cravings at pre-intervention and that this association was significantly weaker at post-intervention (model 1). In contrast, we found that this association did not significantly differ between onemonth follow-up and post-intervention (hypothesis 2, model 2). Thus, training in mindful eating did reduce the positive within-person association between negative mood and food cravings, but this link did not continue to weaken one month after intensive training ended. This suggests there may be room for optimizing the intervention approach to achieve further decline in relation between mood and cravings following training.

According to the NIH-developed ORBIT model (Czajkowski et al., 2015) of developing behavioral health interventions, results reported here represent a key step in the process of behavioral health intervention development. The ORBIT model outlines steps for developing behavioral treatments that include clearly defining intervention targets and conducting preliminary testing to ensure those targets are engaged. This paradigm changed the way behavioral treatments are developed and optimized by emphasizing the analysis of target engagement to determine the mechanisms through which an intervention works before it is tested in more rigorous trials. In these analyses, we found a link between negative mood and food cravings, and further adding to the literature, we found in model 1 that uncoupling this link was a key target engaged by the intervention. Thus, model 1 results suggest that our intervention works in part by targeting the learned cycle between negative mood and food cravings. Future phases of this work should examine change in clinical outcomes, such as weight.

The intervention we tested in these analyses may have been effective in engaging the association between mood and craving through its training in skills for coping with negative emotions (e.g., sadness and anger) and explaining how poor 
coping can be tethered to overeating (Bodell et al., 2019). These skills may involve identifying patterns of eating in response to mood, and encourage coping skills such as meditation, social support, and other stress-reduction strategies. In one such tool called the Stress Test, participants complete a short mindfulness exercise that encourages them to check in with their body to determine if specific symptoms are present. Then, they receive feedback on whether these symptoms are consistent with stress, habit, or homeostatic hunger, and are given a suggestion to address the relevant symptom. As such, individuals disambiguate and "relearn" which specific body and mindstates are indicative of homeostatic vs. hedonic (i.e., eating for pleasure) hunger. By teaching skills to adaptively identify and cope with emotional difficulties, this intervention assists participants with frequent food cravings by helping them to identify automatic, learned craving tendencies when experiencing a negative mood-and to respond appropriately (eating when signaled by homeostatic hunger, and working with cravings and emotions when signaled by hedonic cues; Beccia et al., 2020).

Future intervention refinement efforts might focus on enhancing these lessons to maximally target the mood-cravings link in an adequately powered RCT design. Qualitative data that examines the utility of these modules in reducing the mood-eating link may contribute to future refinement (e.g., Beccia et al., 2020). For instance, to what extent did participants find modules on self-monitoring and existing with cravings helpful in coping adaptively with cravings? Engaging participants in focus groups around this topic is an important future direction. Analyzing target engagement and uncovering the mechanisms that underpin the intervention's effects will allow researchers to hone mindful eating intervention components to yield an optimized treatment. Beccia and colleagues (2020) recently conducted a focus group among women with binge or emotional eating, who participated in a version of the Eat Right Now program. To better understand participants' experiences, they asked questions such as, "How do you learn to exist with triggers for eating rather than acting upon them?" and "How do you know that you have 'shifted gears' (from increasing awareness of one's eating and triggers for eating, to observing emotional outcomes, to learning not to act on emotional triggers)." They found mindful eating empowered participants to make positive choices about food, eating, and coping, without focusing on weight outcomes. This may be one mechanism through which mindful eating training can reduce problematic craving-related eating.

We also hypothesized that mindful eating training would continue to impart benefits after the intervention ended. Results from model 2, however, did not suggest our hypothesized further weakening of this link. Given the length of the intervention relative to how long these eating patterns had been established and repeatedly reinforced, it is not surprising that individuals may show initial gains, yet without a supported engagement, and may not overcome deeper layers of well-grooved habit patterns, preventing further progress. This highlights the need for a consideration of ways to promote improvement after the intervention ends, such as booster or bonus content (Schlup et al., 2009), should an individual wish to further improve their abilities to cope with food cravings. Future research should carefully consider the additional benefit of integrating further intervention components and design trials that allow for evaluation of individual treatment components.

It is notable that the benefits realized at post-intervention held at follow-up as longer term maintenance of behavioral intervention effects is challenging to achieve (Dombrowski et al., 2014; Douketis et al., 2005; Norris et al., 2016). Future research may further optimize maintenance of behavior change following from this intervention by assessing additional intervention components that specifically target maintenance, such as online community forums wherein individuals can provide support to each other (Lord et al., 2018).

This mobile intervention offers many benefits compared to traditional in-person interventions. Notably, the current global COVID-19 pandemic has highlighted the importance of developing mobile interventions for health behavior change (Figueroa \& Aguilera, 2020). Mobile intervention delivery lifts barriers specific to in-person interventions, such as scheduling, travel, and time commitment (Covolo et al., 2017). With rapid adoption of smartphones and diminishing socioeconomic disparities in smartphone ownership (Pew Research Center, 2019), mobile interventions such as the one tested here may hold promise for cost-effective dissemination to remote and underserved populations (Covolo et al., 2017; Syed et al., 2013). In addition, the mobile format is well-suited to be integrated into the daily lives of users, potentially enabling access to behavioral support tools with greater ease and frequency compared to in-person interventions (Covolo et al., 2017; Free et al., 2013). This may be particularly relevant for behaviors that involve repeated, ongoing maintenance to achieve desired health benefits, such as eating behavior, for which individuals may benefit from being able to engage with intervention tools in real time upon recognizing potential triggers of unhealthy eating.

This intervention targets unwanted food cravings and craving-related eating behavior, rather than weight loss and dietary adherence, and therefore may be an important approach for a significant subset of the population with overweight and obesity for whom diet interventions often fail. For example, many individuals may have had failed dieting attempts because of underlying dysregulated eating behavior (cravings) that standard diet interventions did not sufficiently attend to (Franz et al., 2007; Meule et al., 2012, 2017). Mindfulness-based interventions, such as the current one, may be particularly beneficial above and beyond 
standard weight loss programs because they target underlying dysregulated eating behavior (Radin et al., 2020). It is also plausible that the weakening of the mood-craving link could be a mechanism of change among populations with binge or emotional eating, which researchers have posited exist along a continuum (Vainik et al., 2015). Mindfulnessbased eating interventions have shown promise in reducing problematic overeating on the more severe end (binge eating; Godfrey et al., 2015). However, researchers have yet to focus on uncovering treatment mechanisms at varying levels of problematic overeating. These gaps highlight the need to better assess mechanisms of change in mindfulness-based interventions for non-homeostatic eating, such as problematic overeating.

\section{Limitations and Future Research}

These analyses do have limitations that suggest future directions. These analyses included self-report data; however, we used daily-level data (ecological momentary assessment methods) which reduce recall bias. Future work could introduce a behavioral measurement of food cravings, such as presenting food in a laboratory eating paradigm and assessing cravings in the moment as a more objective assessment. We also sent EMA text message prompts to participants at the same time of day each day. Future research could personalize the time surveys are sent, randomize the time surveys are sent, and/or allow participants to initiate survey completion. This would allow participants to complete survey measures in the moment when they experience a negative mood or experiencing a food craving. Our study used a small sample size; however, this approach is aligned with the Science of Behavior Change recommendations for feasibility studies and testing treatment mechanisms (Science of Behavior Change, 2020). Although participants were able to continue using the app after the intervention, we did not track whether participants were practicing mindful eating during the one-month follow-up period or how they were engaging with the app. Finally, our study targeted only one predictor of craving (affect), given strong mechanistic support for an affectcraving association (Haedt-Matt \& Keel, 2011; Heatherton $\&$ Baumeister, 1991). However, there are multiple determinants of non-homeostatic eating such as the availability of highly rewarding, highly processed food (Elliston et al., 2017). Future interventions may identify other predictors and develop assessment and intervention components to optimize treatment. Although our trial was not adequately powered to perform sub-group analyses, future work should seek to explore the moderating role of trait-level eating on the momentary links between affect and cravings.

The present study established a mechanistic affective pathway through which a smartphone-delivered mindful eating intervention reduces food cravings. This theorydriven intervention grounded in an operant conditioning model of eating behavior was effective in engaging negative mood and craving targets and reducing their learned association post-intervention. Although this link did not further weaken between post-intervention and onemonth follow-up, we did see a maintenance effect (lack of change between post-intervention and one-month followup), suggesting that future intervention refinements may be needed to achieve further reductions. Taken together, previous analyses identified feasibility and acceptability of this intervention in a targeted sample with overweight and high in food cravings (Mason et al., 2018) and the present analyses have further clarified treatment mechanisms. Future research should seek to refine the intervention and test the effects in an RCT with an adequately matched control group.

Supplementary Information The online version contains supplementary material available at https://doi.org/10.1007/s12671-021-01760-z.

Author Contribution SJSH: contributed to the study conception and design, prepared the material and collected the data, wrote the first draft of the manuscript, performed data analysis, provided critical feedback and edits to each version of the manuscript, read and approved the final manuscript. RMR: contributed to the study conception and design, performed data analysis, wrote the first draft of the manuscript, provided critical feedback and edits to each version of the manuscript, read and approved the final manuscript. KJ: prepared the material and collected the data, wrote the first draft of the manuscript, provided critical feedback and edits to each version of the manuscript, read and approved the final manuscript. JAB: provided feedback on the study design and manuscript preparation, provided critical feedback and edits to each version of the manuscript, read and approved the final manuscript. MC: prepared the material and collected the data, provided critical feedback and edits to each version of the manuscript, read and approved the final manuscript.

WH: performed the data analysis, provided critical feedback and edits to each version of the manuscript, read and approved the final manuscript. AEM: contributed to the study conception and design, provided critical feedback and edits to each version of the manuscript, read and approved the final manuscript.

Sara J. Sagui-Henson and Rachel M. Radin contributed equally as co-first authors.

Data Availability All data are available at the Open Science Framework (https://osf.io/ud92x/).

\section{Declarations}

Ethics Approval This study was approved by the University of California San Francisco (UCSF) Institutional Review Board (IRB) and was therefore performed in accordance with the ethical standards laid down in the 1964 Declaration of Helsinki and its later amendments.

Informed Consent All participants provided written informed consent prior to their inclusion in the study.

Conflict of Interest Author Brewer owns stock in and serves as a paid consultant for Sharecare Inc., the company that owns the mindfulness 
app used in this study. Authort Brewer's financial interest has been disclosed to and is being managed by Brown University, in accordance with its Conflict of Interest and Conflict of Commitment policies. All other authors declare no competing interests.

\section{References}

Abdella, H., El Farssi, H., Broom, D., Hadden, D., \& Dalton, C. (2019). Eating behaviours and food cravings: Influence of age, sex. BMI and FTO Genotype. Nutrients, 11(2), 377. https://doi.org/10.3390/ nu11020377

Agras, W. S., \& Telch, C. F. (1998). The effects of caloric deprivation and negative affect on binge eating in obese binge-eating disordered women. Behavior Therapy, 29(3), 491-503. https://doi.org/ 10.1016/S0005-7894(98)80045-2

Arnow, B., Kenardy, J., \& Agras, W. S. (1995). The emotional eating scale: The development of a measure to assess coping with negative affect by eating. International Journal of Eating Disorders, 18(1), 79-90. https://doi.org/10.1002/1098-108X(199507)18:1\% 3c79::AID-EAT2260180109\%3e3.0.CO;2-V

Barnhart, W. R., Braden, A. L., \& Dial, L. (2021). Understanding the relationship between negative emotional eating and binge eating: The moderating effects of acting with awareness and non-reactive mindfulness. Journal of Clinical Psychology, 77, 1954-1972. https://doi.org/10.1002/jclp.23123

Beccia, A. L., Ruf, A., Druker, S., Ludwig, V. U., \& Brewer, J. A. (2020). Women's experiences with a mindful eating program for binge and emotional eating: A qualitative investigation into the process of change. The Journal of Alternative and Complementary Medicine, 26(10), 937-944. https://doi.org/10.1089/acm. 2019.0318

Beshara, M., Hutchinson, A. D., \& Wilson, C. (2013). Does mindfulness matter? Everyday mindfulness, mindful eating and selfreported serving size of energy dense foods among a sample of South Australian adults. Appetite, 67, 25-29. https://doi.org/10. 1016/j.appet.2013.03.012

Bodell, L. P., Pearson, C. M., Smith, K. E., Cao, L., Crosby, R. D., Peterson, C. B., Crow, S. J., \& Berg, K. C. (2019). Longitudinal associations between emotion regulation skills, negative affect, and eating disorder symptoms in a clinical sample of individuals with binge eating. Eating Behaviors, 32, 69-73. https://doi.org/ 10.1016/j.eatbeh.2018.12.005

Bolger, N., \& Laurenceau, J.-P. (2013). Intensive longitudinal methods: An introduction to diary and experience sampling research (1st ed.). Guilford Press.

Bouton, M. E. (2011). Learning and the persistence of appetite: Extinction and the motivation to eat and overeat. Physiology \& Behavior, 103(1), 51-58. https://doi.org/10.1016/j.physbeh.2010.11.025

Brewer, J. A., Ruf, A., Beccia, A. L., Essien, G. I., Finn, L. M., van Lutterveld, R., \& Mason, A. E. (2018). Can mindfulness address maladaptive eating behaviors? Why traditional diet plans fail and how new mechanistic insights may lead to novel interventions. Frontiers in Psychology, 9, 1418. https://doi.org/10.3389/fpsyg. 2018.01418

Brown, K. W., Ryan, R. M., \& Creswell, J. D. (2007). Mindfulness: Theoretical foundations and evidence for its salutary effects. Psychological Inquiry, 18(4), 211-237. https://doi.org/10.1080/10478 400701598298

Carels, R. A., Hoffman, J., Collins, A., Raber, A. C., Cacciapaglia, H., \& O'Brien, W. H. (2001). Ecological momentary assessment of temptation and lapse in dieting. Eating Behaviors, 2(4), 307-321. https://doi.org/10.1016/S1471-0153(01)00037-X

Chao, A., Grilo, C. M., White, M. A., \& Sinha, R. (2014). Food cravings, food intake, and weight status in a community-based sample.
Eating Behaviors, 15(3), 478-482. https://doi.org/10.1016/j.eatbeh.2014.06.003

Corsica, J. A., \& Spring, B. J. (2008). Carbohydrate craving: A doubleblind, placebo-controlled test of the self-medication hypothesis. Eating Behaviors, 9(4), 447-454. https://doi.org/10.1016/j.eatbeh. 2008.07.004

Covolo, L., Ceretti, E., Moneda, M., Castaldi, S., \& Gelatti, U. (2017). Does evidence support the use of mobile phone apps as a driver for promoting healthy lifestyles from a public health perspective? A systematic review of randomized control trials. Patient Education and Counseling, 100(12), 2231-2243. https://doi.org/10. 1016/j.pec.2017.07.032

Czajkowski, S. M., Powell, L. H., Adler, N., Naar-King, S., Reynolds, K. D., Hunter, C. M., Laraia, B., Olster, D. H., Perna, F. M., Peterson, J. C., Epel, E., Boyington, J. E., \& Charlson, M. E. (2015). From ideas to efficacy: The ORBIT model for developing behavioral treatments for chronic diseases. Health Psychology, 34(10), 971-982. https://doi.org/10.1037/hea0000161

Dalton, M., Blundell, J., \& Finlayson, G. S. (2013). Examination of food reward and energy intake under laboratory and free-living conditions in a trait binge eating subtype of obesity. Frontiers in Psychology, 4. https://doi.org/10.3389/fpsyg.2013.00757

Daubenmier, J., Moran, P. J., Kristeller, J., Acree, M., Bacchetti, P., Kemeny, M. E., Dallman, M., Lustig, R. H., Grunfeld, C., Nixon, D. F., Milush, J. M., Goldman, V., Laraia, B., Laugero, K. D., Woodhouse, L., Epel, E. S., \& Hecht, F. M. (2016). Effects of a mindfulness-based weight loss intervention in adults with obesity: A randomized clinical trial: Mindfulness-Based Weight Loss for Obesity. Obesity, 24(4), 794-804. https://doi.org/10.1002/oby. 21396

Delahanty, L. M., Meigs, J. B., Hayden, D., Williamson, D. A., \& Nathan, D. M. (2002). Psychological and behavioral correlates of baseline BMI in the Diabetes Prevention Program (DPP). Diabetes Care, 25(11), 1992-1998. https://doi.org/10.2337/diacare. 25.11.1992

Dombrowski, S. U., Knittle, K., Avenell, A., Araujo-Soares, V., \& Sniehotta, F. F. (2014). Long term maintenance of weight loss with non-surgical interventions in obese adults: Systematic review and meta-analyses of randomised controlled trials. BMJ, 348(may 14 6), g2646-g2646. https://doi.org/10.1136/bmj.g2646

Douketis, J. D., Macie, C., Thabane, L., \& Williamson, D. F. (2005). Systematic review of long-term weight loss studies in obese adults: Clinical significance and applicability to clinical practice. International Journal of Obesity, 29(10), 1153-1167. https://doi. org/10.1038/sj.ijo.0802982

Elfhag, K., \& Rossner, S. (2005). Who succeeds in maintaining weight loss? A conceptual review of factors associated with weight loss maintenance and weight regain. Obesity Reviews, 6(1), 67-85. https://doi.org/10.1111/j.1467-789X.2005.00170.x

Elliston, K. G., Ferguson, S. G., Schüz, N., \& Schüz, B. (2017). Situational cues and momentary food environment predict everyday eating behavior in adults with overweight and obesity. Health Psychology, 36(4), 337. https://doi.org/10.1037/hea0000439

Epstein, L. H., Leddy, J. J., Temple, J. L., \& Faith, M. S. (2007). Food reinforcement and eating: A multilevel analysis. Psychological Bulletin, 133(5), 884-906. https://doi.org/10.1037/0033-2909. 133.5.884

Falbe, J., Thompson, H. R., Patel, A., \& Madsen, K. A. (2019). Potentially addictive properties of sugar-sweetened beverages among adolescents. Appetite, 133, 130-137. https://doi.org/10.1016/j. appet.2018.10.032

Figueroa, C. A., \& Aguilera, A. (2020). The need for a mental health technology revolution in the COVID-19 pandemic. Frontiers in Psychiatry, 11(523). https://doi.org/10.3389/fpsyt.2020.00523

Franz, M. J., VanWormer, J. J., Crain, A. L., Boucher, J. L., Histon, T., Caplan, W., Bowman, J. D., \& Pronk, N. P. (2007). Weight-loss 
outcomes: A systematic review and meta-analysis of weight-loss clinical trials with a minimum 1-year follow-up. Journal of the American Dietetic Association, 107(10), 1755-1767. https://doi. org/10.1016/j.jada.2007.07.017

Free, C., Phillips, G., Galli, L., Watson, L., Felix, L., Edwards, P., Patel, V., \& Haines, A. (2013). The effectiveness of mobilehealth technology-based health behaviour change or disease management interventions for health care consumers: A systematic review. PLoS Medicine, 10(1), e1001362. https://doi. org/10.1371/journal.pmed.1001362

Godfrey, K. M., Gallo, L. C., \& Afari, N. (2015). Mindfulness-based interventions for binge eating: A systematic review and metaanalysis. Journal of Behavioral Medicine, 38(2), 348-362. https://doi.org/10.1007/s10865-014-9610-5

Haedt-Matt, A. A., \& Keel, P. K. (2011). Revisiting the affect regulation model of binge eating: A meta-analysis of studies using ecological momentary assessment. Psychological Bulletin, 137(4), 660-681. https://doi.org/10.1037/a0023660

Heatherton, T. F., \& Baumeister, R. F. (1991). Binge eating as escape from self-awareness. Psychological Bulletin, 110(1), 86-108. https://doi.org/10.1037/0033-2909.110.1.86

Hetherington, M. M., \& MacDiarmid, J. I. (1993). "Chocolate addiction": A preliminary study of its description and its relationship to problem eating. Appetite, 21(3), 233-246. https://doi.org/10. 1006/appe. 1993.1042

Hilbert, A., \& Tuschen-Caffier, B. (2007). Maintenance of binge eating through negative mood: A naturalistic comparison of binge eating disorder and bulimia nervosa. The International Journal of Eating Disorders, 40(6), 521-530. https://doi.org/ 10.1002/eat.20401

Hill, A. J., \& Heaton-Brown, L. (1994). The experience of food craving: A prospective investigation in healthy women. Journal of Psychosomatic Research, 38(8), 801-814. https://doi.org/10.1016/ 0022-3999(94)90068-X

Hill, A. J., Weaver, C. F. L., \& Blundell, J. E. (1991). Food craving, dietary restraint and mood. Appetite, 17(3), 187-197. https://doi. org/10.1016/0195-6663(91)90021-J

Hoge, E. A., Bui, E., Marques, L., Metcalf, C. A., Morris, L. K., Robinaugh, D. J., Worthington, J. J., Pollack, M. H., \& Simon, N. M. (2013). Randomized controlled trial of mindfulness meditation for generalized anxiety disorder: Effects on anxiety and stress reactivity. The Journal of Clinical Psychiatry, 74(8), 786-792. https:// doi.org/10.4088/JCP.12m08083

IBM Corp. (2017). IBM Statistics for Windows, Version 25.0. IBM Corp.

Jordan, C. H., Wang, W., Donatoni, L., \& Meier, B. P. (2014). Mindful eating: Trait and state mindfulness predict healthier eating behavior. Personality and Individual Differences, 68, 107-111. https://doi.org/10.1016/j.paid.2014.04.013

Kristeller, J. L., \& Hallett, C. B. (1999). An exploratory study of a meditation-based intervention for binge eating disorder. Journal of Health Psychology, 4(3), 357-363. https://doi.org/10.1177/13591 0539900400305

Kristeller, J. L., \& Wolever, R. Q. (2010). Mindfulness-based eating awareness training for treating binge eating disorder: The conceptual foundation. Eating Disorders, 19(1), 49-61. https://doi.org/ 10.1080/10640266.2011.533605

Kristeller, J. L., Wolever, R. Q., \& Sheets, V. (2014). Mindfulnessbased eating awareness training (MB-EAT) for binge eating: A randomized clinical trial. Mindfulness, 5(3), 282-297. https://doi. org/10.1007/s12671-012-0179-1

Littell, R. C., Milliken, G. A., Stroup, W. W., Wolfinger, R. D., \& Schabenberger, O. (2006). SAS for mixed models, Second Edition. SAS Institute.

Little, R. J. A. (1988). A test of missing completely at random for multivariate data with missing values. Journal of the American
Statistical Association, 83(404), 1198-1202. https://doi.org/10. 1080/01621459.1988.10478722

Lord, V. M., Reiboldt, W., Gonitzke, D., Parker, E., \& Peterson, C. (2018). Experiences of recovery in binge-eating disorder: A qualitative approach using online message boards. Eating and Weight Disorders-Studies on Anorexia, Bulimia and Obesity, 23(1), 95-105. https://doi.org/10.1007/s40519-016-0335-z

Mason, A. E., Epel, E. S., Aschbacher, K., Lustig, R. H., Acree, M., Kristeller, J., Cohn, M., Dallman, M., Moran, P. J., Bacchetti, P., Laraia, B., Hecht, F. M., \& Daubenmier, J. (2016a). Reduced reward-driven eating accounts for the impact of a mindfulnessbased diet and exercise intervention on weight loss: Data from the SHINE randomized controlled trial. Appetite, 100, 86-93. https:// doi.org/10.1016/j.appet.2016.02.009

Mason, A. E., Epel, E. S., Kristeller, J., Moran, P. J., Dallman, M., Lustig, R. H., Acree, M., Bacchetti, P., Laraia, B. A., \& Hecht, F. M. (2016b). Effects of a mindfulness-based intervention on mindful eating, sweets consumption, and fasting glucose levels in obese adults: Data from the SHINE randomized controlled trial. Journal of Behavioral Medicine, 39(2), 201-213. https://doi.org/ 10.1007/s10865-015-9692-8

Mason, A. E., Jhaveri, K., Cohn, M., \& Brewer, J. A. (2018). Testing a mobile mindful eating intervention targeting craving-related eating: Feasibility and proof of concept. Journal of Behavioral Medicine, 41(2), 160-173. https://doi.org/10.1007/s10865-017-9884-5

Massey, A., \& Hill, A. J. (2012). Dieting and food craving: A descriptive, quasi-prospective study. Appetite, 58(3), 781-785. https:// doi.org/10.1016/j.appet.2012.01.020

Meule, A., Lutz, A., Vögele, C., \& Kübler, A. (2012). Food cravings discriminate differentially between successful and unsuccessful dieters and non-dieters: Validation of the Food Cravings Questionnaires in German. Appetite, 58(1), 88-97. https://doi.org/10. 1016/j.appet.2011.09.010

Meule, A., Richard, A., \& Platte, P. (2017). Food cravings prospectively predict decreases in perceived self-regulatory success in dieting. Eating Behaviors, 24, 34-38. https://doi.org/10.1016/j. eatbeh.2016.11.007

Munsch, S., Meyer, A. H., Quartier, V., \& Wilhelm, F. H. (2012). Binge eating in binge eating disorder: A breakdown of emotion regulatory process? Psychiatry Research, 195(3), 118-124. https://doi. org/10.1016/j.psychres.2011.07.016

Ng, L., \& Davis, C. (2013). Cravings and food consumption in binge eating disorder. Eating Behaviors, 14(4), 472-475. https://doi.org/ 10.1016/j.eatbeh.2013.08.011

Norris, S. L., Zhang, X., Avenell, A., Gregg, E., Brown, T., Schmid, C. H., \& Lau, J. (2016). Long-term non-pharmacological weight loss interventions for adults with type 2 diabetes mellitus. Sao Paulo Medical Journal, 134(2), 184-184. https://doi.org/10.1590/ 1516-3180.20161342T2

Pew Research Center. (2019). Demographics of mobile device ownership and adoption in the United States. Pew Research Center: Internet, Science \& Tech. https://www.pewresearch.org/internet/ fact-sheet/mobile/

Polivy, J., \& Herman, C. P. (1993). Etiology of binge eating: Psychological mechanisms. In G. C. Fairburn \& G. T. Wilson (Eds.), Binge eating: Nature, assessment, and treatment (pp. 173-205). Guilford Press.

Radin, R. M., Epel, E. S., Daubenmier, J., Moran, P., Schleicher, S., Kristeller, J., Hecht, F. M., \& Mason, A. E. (2020). Do stress eating or compulsive eating influence metabolic health in a mindfulness-based weight loss intervention? Health Psychology, 39(2), 147-158. https://doi.org/10.1037/hea0000807

Sala, M., Ram, S. S., Vanzhula, I. A., \& Levinson, C. A. (2020). Mindfulness and eating disorder psychopathology: A meta-analysis. International Journal of Eating Disorders, 53(6), 834-851. https://doi.org/10.1002/eat.23247 
Schlup, B., Munsch, S., Meyer, A. H., Margraf, J., \& Wilhelm, F. H. (2009). The efficacy of a short version of a cognitive-behavioral treatment followed by booster sessions for binge eating disorder. Behaviour Research and Therapy, 47(7), 628-635. https://doi.org/ 10.1016/j.brat.2009.04.003

Science of Behavior Change. (2020). Science of behavior change. https://scienceofbehaviorchange.org/

Shiffman, S., Stone, A. A., \& Hufford, M. R. (2008). Ecological momentary assessment. Annual Review of Clinical Psychology, 4, 1-32. https://doi.org/10.1146/annurev.clinpsy.3.022806.091415

Singer, J. D., \& Willett, J. B. (2003). Applied longitudinal data analysis: Modeling change and event occurrence. Oxford University Press.

Singh, M. (2014). Mood, food, and obesity. Frontiers in Psychology, 5. https://doi.org/10.3389/fpsyg.2014.00925

Skinner, B. F. (1963). Operant behavior. American Psychologist, 18(8), 503-515. https://doi.org/10.1037/h0045185

Smyth, J. M., Wonderlich, S. A., Sliwinski, M. J., Crosby, R. D., Engel, S. G., Mitchell, J. E., \& Calogero, R. M. (2009). Ecological momentary assessment of affect, stress, and binge-purge behaviors: Day of week and time of day effects in the natural environment. International Journal of Eating Disorders, 42(5), 429-436. https://doi.org/10.1002/eat.20623

Stone, A. A., \& Shiffman, S. (1994). Ecological momentary assessment (EMA) in behavorial medicine. Annals of Behavioral Medicine, 16(3), 199-202. https://doi.org/10.1093/abm/16.3.199

Syed, S. T., Gerber, B. S., \& Sharp, L. K. (2013). Traveling towards disease: Transportation barriers to health care access. Journal of Community Health, 38(5), 976-993. https://doi.org/10.1007/ s10900-013-9681-1

Telch, C. F., \& Agras, W. S. (1996). Do emotional states influence binge eating in the obese? International Journal of Eating
Disorders, 20(3), 271-279. https://doi.org/10.1002/(SICI)1098108X(199611)20:3\%3c271::AID-EAT6\%3e3.0.CO;2-L

Vainik, U., Neseliler, S., Konstabel, K., Fellows, L. K., \& Dagher, A. (2015). Eating traits questionnaires as a continuum of a single concept. Uncontrolled Eating. Appetite, 90, 229-239. https://doi. org/10.1016/j.appet.2015.03.004

Ventura, T., Santander, J., Torres, R., \& Contreras, A. M. (2014). Neurobiologic basis of craving for carbohydrates. Nutrition, 30(3), 252-256. https://doi.org/10.1016/j.nut.2013.06.010

Wegner, K. E., Smyth, J. M., Crosby, R. D., Wittrock, D., Wonderlich, S. A., \& Mitchell, J. E. (2002). An evaluation of the relationship between mood and binge eating in the natural environment using ecological momentary assessment. International Journal of Eating Disorders, 32(3), 352-361. https://doi.org/10.1002/eat.10086

Weingarten, H. P., \& Elston, D. (1990). The phenomenology of food cravings. Appetite, 15(3), 231-246. https://doi.org/10.1016/01956663(90)90023-2

Whiteside, U., Chen, E., Neighbors, C., Hunter, D., Lo, T., \& Larimer, M. (2007). Difficulties regulating emotions: Do binge eaters have fewer strategies to modulate and tolerate negative affect? Eating Behaviors, 8(2), 162-169. https://doi.org/10.1016/j.eatbeh.2006. 04.001

Woolhouse, H., Knowles, A., \& Crafti, N. (2012). Adding mindfulness to CBT programs for binge eating: A mixed-methods evaluation. Eating Disorders, 20(4), 321-339. https://doi.org/10.1080/10640 266.2012.691791

Publisher's Note Springer Nature remains neutral with regard to jurisdictional claims in published maps and institutional affiliations. 